\title{
Diseño por simulación de un control Fuzzy y MPC para un proceso de nivel
}

\author{
Edison D. Mañay \\ edmanay@espe.edu.ec \\ https://orcid.org/0000-0002-3447-2511 \\ Universidad de las Fuerzas Armadas ESPE
}

Mauricio D. Chiliquinga

mdchiliquinga@espe.edu.ec

Universidad de las Fuerzas Armadas ESPE

Johanna E. Iza lza

jeiza1@espe.edu.ec

Universidad de las Fuerzas Armadas ESPE

\author{
Héctor Efraín Yanchatipán Ugsha \\ hector.yanchatipan8728@utc.edu.ec \\ Universidad Técnica de Cotopaxi \\ Latacunga - Ecuador
}

Paul Salazar Castillo

paulsalazarc91@gmail.com

ISPIB Martha Bucaram de Roldós

Ambato - Ecuador

\section{RESUMEN}

El artículo presenta la evaluación de un control Fuzzy y MPC para un proceso de nivel utilizando la herramienta Simulink de Matlab. El problema más frecuente en la industria de procesos es el control de nivel en tanques ya que presenta un modelo matemático no lineal. Comúnmente se utiliza controladores tradicionales como PID para la regulación del nivel en estos sistemas. Para la investigación se considera un tanque cilíndrico caracterizado por una función matemática no lineal. La salida del sistema está definida por $h$ que es el nivel del líquido, que cambia con la apertura de la válvula de entrada $a_{1}$, esta válvula cambia de posición de 0 a 1 (0 a $100 \%)$ y $a_{2}$ representa la válvula de perturbación. Se implementa en Simulink un control en lazo abierto con el fin de verificar la dinámica de la planta para obtener el modelo matemático en espacio de estados, luego 
se diseña el control Fuzzy y el control basado en modelo MPC. Finalmente se contrastan los resultados de los controladores diseñados Fuzzy y MPC con el control tradicional PID, siendo el control Fuzzy: PD difuso con acción integral, presento un mejor rendimiento para el proceso de nivel implementado, ya que, el tiempo de establecimiento es de 119.407 segundos y ante una perturbación se estabiliza en un tiempo de 82.414 segundos y no presenta un sobre impulso.

Palabras clave: proceso de nivel, control fuzzy, MPC, control PID. 


\title{
Design by simulation of a Fuzzy control and MPC \\ for a level process
}

\begin{abstract}
The paper presents the evaluation of a Fuzzy and MPC control for a level process using the Matlab tool Simulink. The most frequent problem in the process industry is the level control in tanks since it presents a nonlinear mathematical model. Traditional controllers such as PID are commonly used for level control in these systems. For the investigation, a cylindrical tank characterized by a nonlinear mathematical function is considered. The output of the system is defined by $h$ which is the liquid level, which changes with the opening of the inlet valve $a_{1}$, this valve changes position from 0 to 1 (0 to $100 \%$ ) and $a_{2}$ represents the disturbance valve. An open loop control is implemented in Simulink in order to verify the dynamics of the plant to obtain the mathematical model in state space, then the Fuzzy control and the control based on MPC model are designed. Finally, the results of the designed Fuzzy and MPC controllers are contrasted with the traditional PID control, being the Fuzzy control: Fuzzy PD with integral action, presented a better performance for the implemented level process, since, the establishment time is 119.407 seconds and before a disturbance it stabilizes in a time of 82.414 seconds and it does not present an over impulse.
\end{abstract}

Keywords: level process; fuzzy control; MPC control; PID control.

Artículo recibido: 30 noviembre. 2021 Aceptado para publicación: 29 diciembre 2021

Correspondencia: edmanay@espe.edu.ec Conflictos de Interés: Ninguna que declarar 


\section{INTRODUCCIÓN}

En la industria de pintura, metalurgia, alimentos, farmacéuticas, tratamiento de aguas y refinerías de petróleo, el proceso de medición y control del nivel de líquido es muy importante. Si nos enfocamos en el aspecto económico de las empresas, el contenido de los productos debe ser exactas y de la misma forma si no hay la correcta medida tanto la empresa y el producto pierde credibilidad. También al hablar del llenado de un tanque, está directamente inmerso la altura (h), que el controlador procura mantener en el punto de ajuste deseado y disminuir el error, busca extender la vida útil de los actuadores. Existen controladores clásicos y modernos, como PID, Fuzzy, MPC, etc., en el presente artículo se diseñan dichos controladores para el proceso de nivel. En la Figura 1, se presenta el plano del proceso de nivel.

\section{Figura 1}

Plano P\&ID de proceso de nivel en un tanque

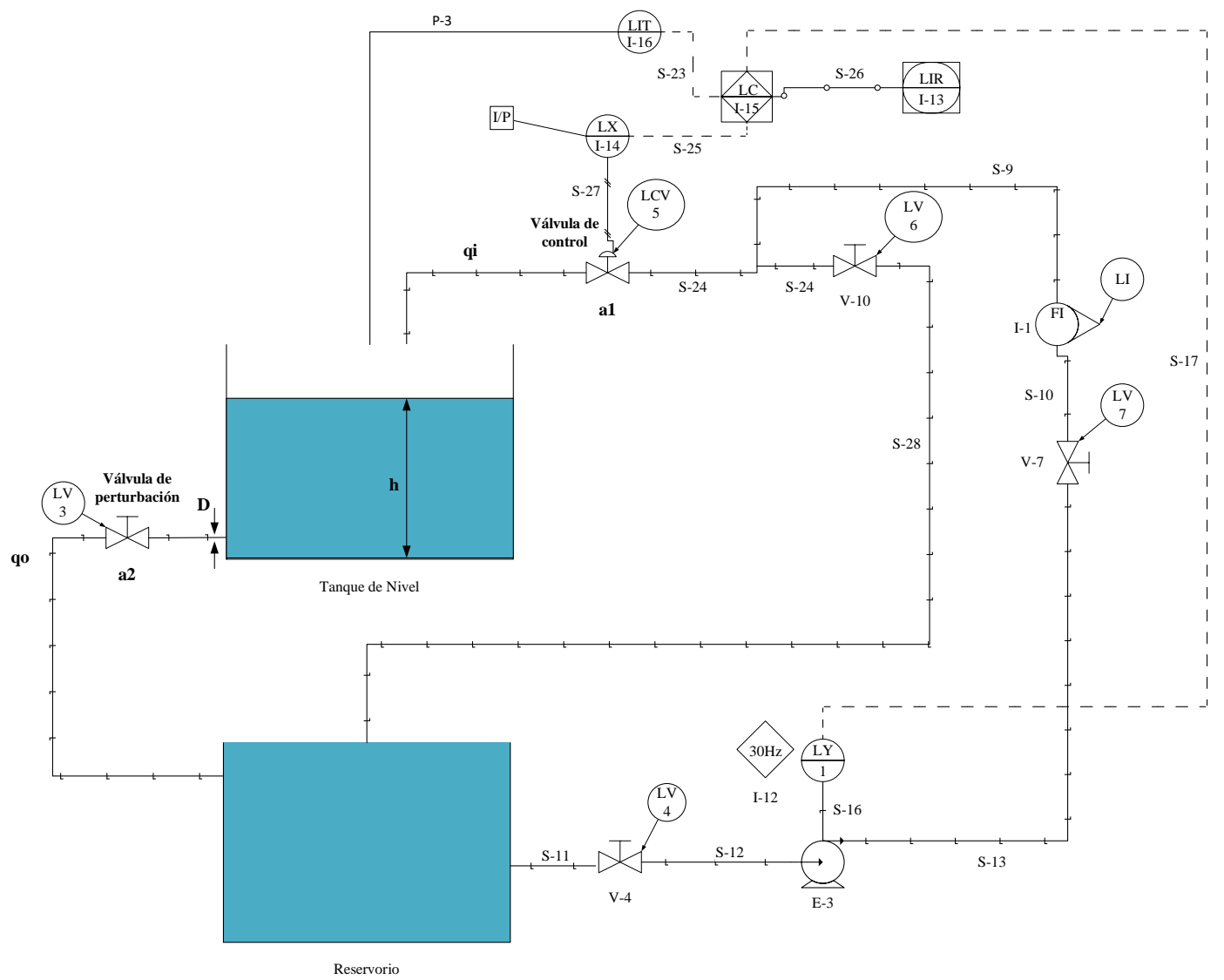

El objetivo es diseñar un controlador por simulación para mantener el nivel del líquido en un punto de ajuste por medio del control del actuador. En las secciones siguientes del artículo, se realiza un análisis del comportamiento dinámico del proceso de nivel en lazo 
abierto, se diseña por simulación un control Fuzzy y un control predictivo basado en modelos (MPC). Finalmente se realiza un análisis del comportamiento del sistema de control Fuzzy, MPC y PID.

\section{Control PID}

El control del nivel del líquido, es necesario en muchos procesos industriales (Alotaibi et al., 2019). Debido a las no linealidades e incertidumbres del proceso, el sistema de control de nivel es bastante complejo. Normalmente, se utilizan controladores PID (o PI) para el control de nivel (Popescu, 2018). Sin embargo, en algunas situaciones, los controladores PID o PI no funcionan correctamente debido a las no linealidades. Para este sistema no lineal, a veces los enfoques de control convencionales no son adecuados.

\section{Control difuso (Fuzzy)}

El controlador de lógica difusa (Fuzzy), es una de las técnicas de control más utilizadas en los procesos industriales. La principal ventaja del controlador difuso es que proporciona un mejor rendimiento que el control convencional en términos de tiempo de establecimiento, tiempo de respuesta, sobreimpulso y robustez (Prusty et al., 2014). El controlador difuso es también muy fácil de implementar, por lo que el controlador puede ser añadido al controlador PID existente (Tzafestas y Papanikolopoulos, 1990). Sin embargo, el controlador difuso no puede eliminar el error en estado estacionario, mientras que los controladores PID tiene la capacidad de eliminar los errores de estado estacionario (Prusty et al., 2014). Por lo tanto, cuando se conjuga los dos controladores son capaz de controlar el proceso de mejor manera (Mursyitah et al., 2018).

\section{Control predictivo basado en modelo (MPC)}

El MPC es una de las técnicas avanzadas de control de procesos (De Prada, 2010). El MPC predice los valores de salida futuros con la ayuda del comportamiento actual y pasado. Utiliza un modelo de sistema para calcular una señal de salida en un horizonte de predicción en el futuro (Gehlaut et al., 2018). El MPC no sólo mejora el rendimiento del control del proceso industrial, sino que también se ocupa de las restricciones y puede utilizarse con un sistema de múltiples entradas y salidas (MIMO) (Troncoso y Suárez, 2017). La señal de control se calcula minimizando una función de coste (Suwannik et al., 2016). La estructura básica del control MPC, se ilustra en la Figura 2. 


\section{Figura 2}

Estructura del control MPC

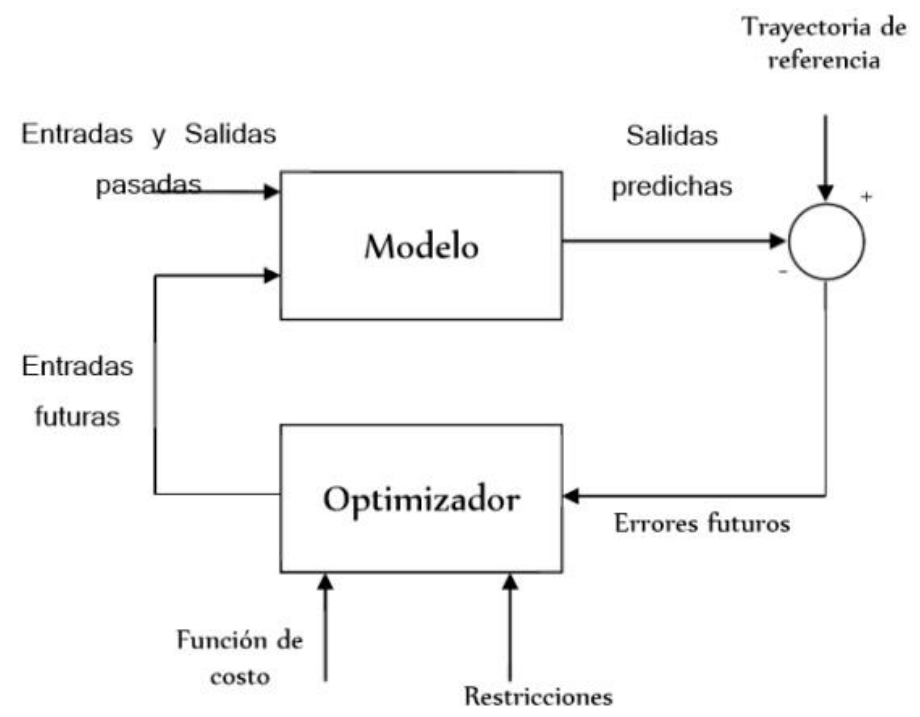

\section{MATERIALES Y MÉTODOS}

El proceso de control del nivel de líquido se muestra en la Figura 3, donde el objetivo es mantener el nivel de líquido del tanque en una altura deseada.

\section{Figura 3}

Planta de nivel de un tanque

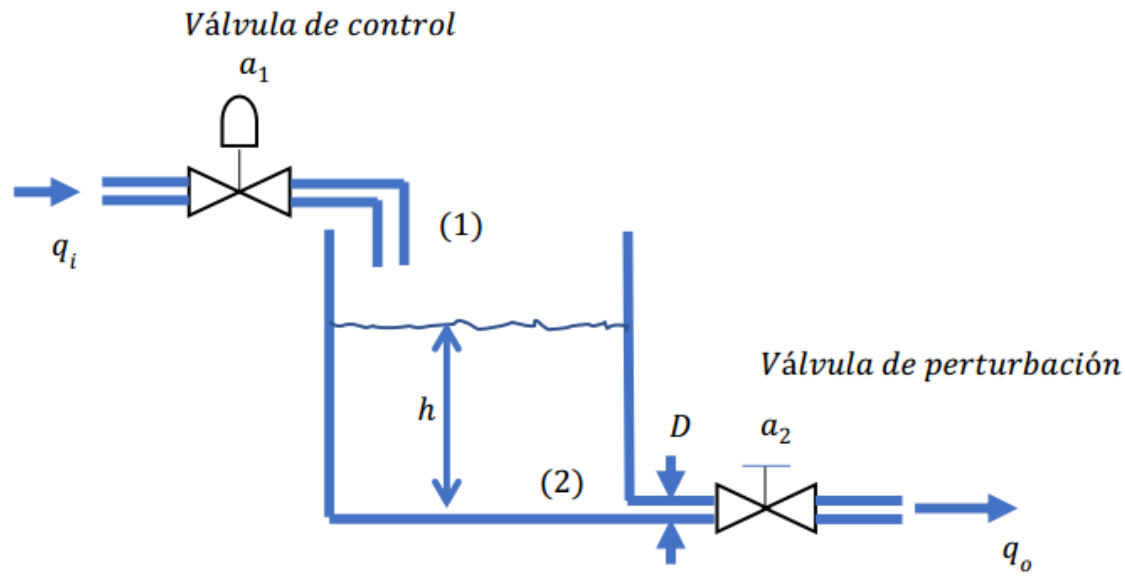

La representación matemática del proceso de nivel de la Figura 3, representada por un modelo no lineal (Åström y Murray, 2008), se representa en la ecuación 1:

$A \frac{d h(t)}{d t}=k_{1} a_{1}-k_{2} a_{2} \sqrt{2 g h(t)}$

Se analiza la planta con el modelo matemático no lineal, Ecuación 1. La Figura 3, representa una planta de nivel de un tanque cilíndrico el cual cuenta con los siguientes 
datos: diámetro de $D_{c}=1 \mathrm{~m}$, radio del cilindro $r=0.5 \mathrm{~m}$. Entonces el área del tanque es calculada $A=\pi(0.5)^{2} ; A=0.785 \mathrm{~m}^{2}$. Se conoce que la gravedad $g=9,8 \mathrm{~s} / \mathrm{m}^{2}$. Las constantes $k 1=0,05$ y $k 2=0,02, a_{1}$ varia de $(0$ a 1$)$ y $a_{2}=0,4$ inicialmente.

Con los parámetros de la planta de nivel establecidos, se implementa la simulación a lazo abierto en Simulink, como se muestra en la Figura 4.

\section{Figura 4}

Sistema de control a lazo abierto

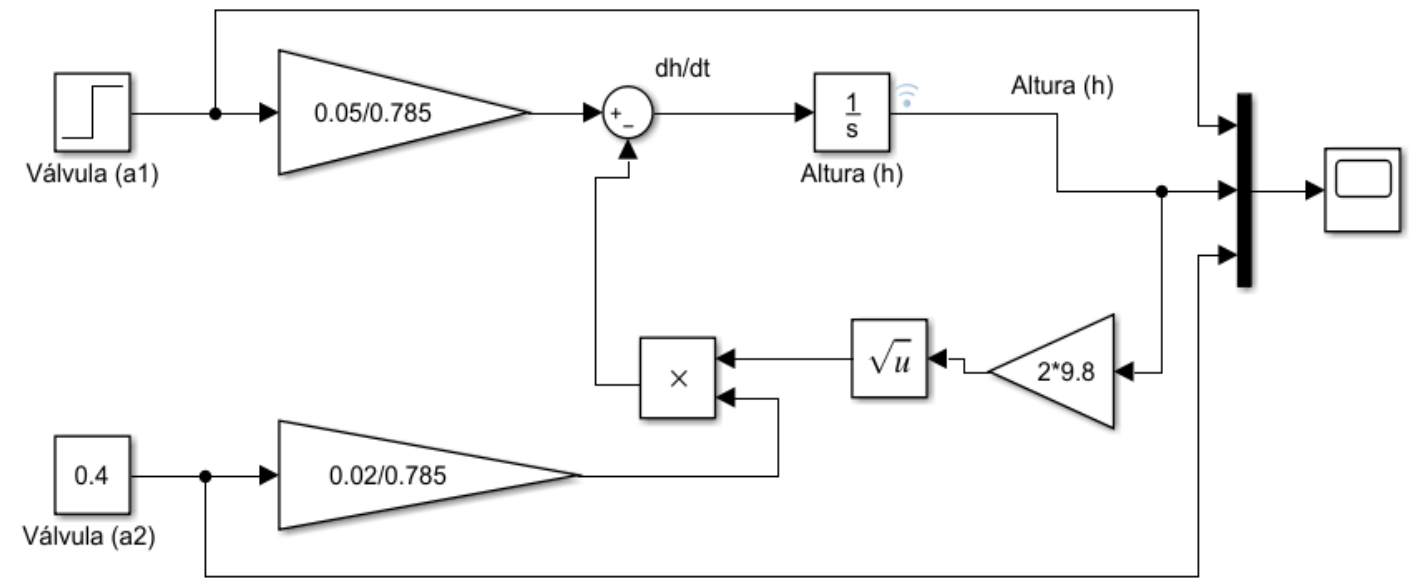

La Figura 4, se manipula a la válvula $a_{1}$ en pasos de 0.1 (10\%) por medio de la entrada tipo escalón y la válvula $a_{2}$ se mantiene con una apertura de 0.4 (40\%) para verificar la salida representada por la altura $h$ en Simulink (Pérez et al., 2008). En la Figura 5, se puede visualizar los resultados y en la Tabla 1 , los valores obtenidos.

\section{Figura 5}

Comportamiento del sistema a lazo abierto con diferentes valores de entradas

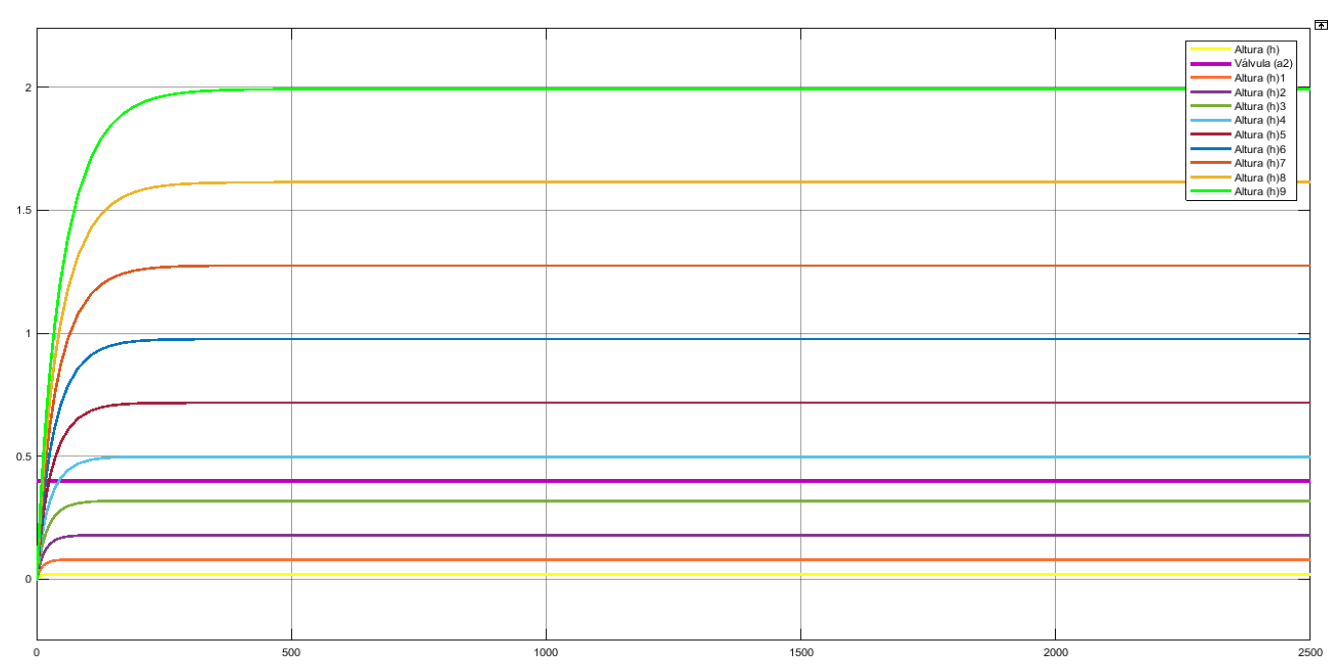




\section{Tabla 1}

Valores de entrada vs. salida del control en lazo abierto

\begin{tabular}{cccc}
\hline $\begin{array}{c}\text { Apertura } \\
\text { Válvula (a1) }\end{array}$ & $\begin{array}{c}\text { Apertura } \\
\text { Válvula (a2) }\end{array}$ & $\begin{array}{c}\text { Nivel del líquido } \\
\text { (h) }\end{array}$ & $\begin{array}{c}\text { Tiempo de } \\
\text { establecimiento } \\
\text { (segundos) }\end{array}$ \\
\hline 0.1 & 0.4 & 0.01973 & 34 \\
0.2 & 0.4 & 0.07892 & 86 \\
0.3 & 0.4 & 0.1776 & 151 \\
0.4 & 0.4 & 0.3157 & 180 \\
0.5 & 0.4 & 0.4933 & 251 \\
0.6 & 0.4 & 0.7103 & 300 \\
0.7 & 0.4 & 0.9668 & 334 \\
0.8 & 0.4 & 1.263 & 370 \\
0.9 & 0.4 & 1.598 & 374 \\
1 & 0.4 & 1.973 & 425 \\
\hline
\end{tabular}

La Tabla 1, refleja una clara desviación del nivel $h$ con respecto a la entrada válvula $a_{1}$, ya que no realizamos ninguna acción de control. Se establece que la altura máxima que puede alcanzar es 1.973 cuando la válvula $a_{1}$ se encuentra abierta al $100 \%$.

Luego de verificar el comportamiento dinámico de la planta en laso abierto mediante un modelo matemático no lineal en Simulink, se procede a obtener un modelo que represente el proceso en espacio de estados, se requiere obtener un conjunto de datos de las entradas y salidas, consiste en aplicar diferentes señales a la entrada de la planta para verificar la evolución del proceso a lo largo del tiempo (salida), los mismos que son almacenados para su posterior análisis. El esquema implementado se visualiza en la Figura 6.

\section{Figura 6}

Sistema de control a lazo abierto para identificación de modelo matemático

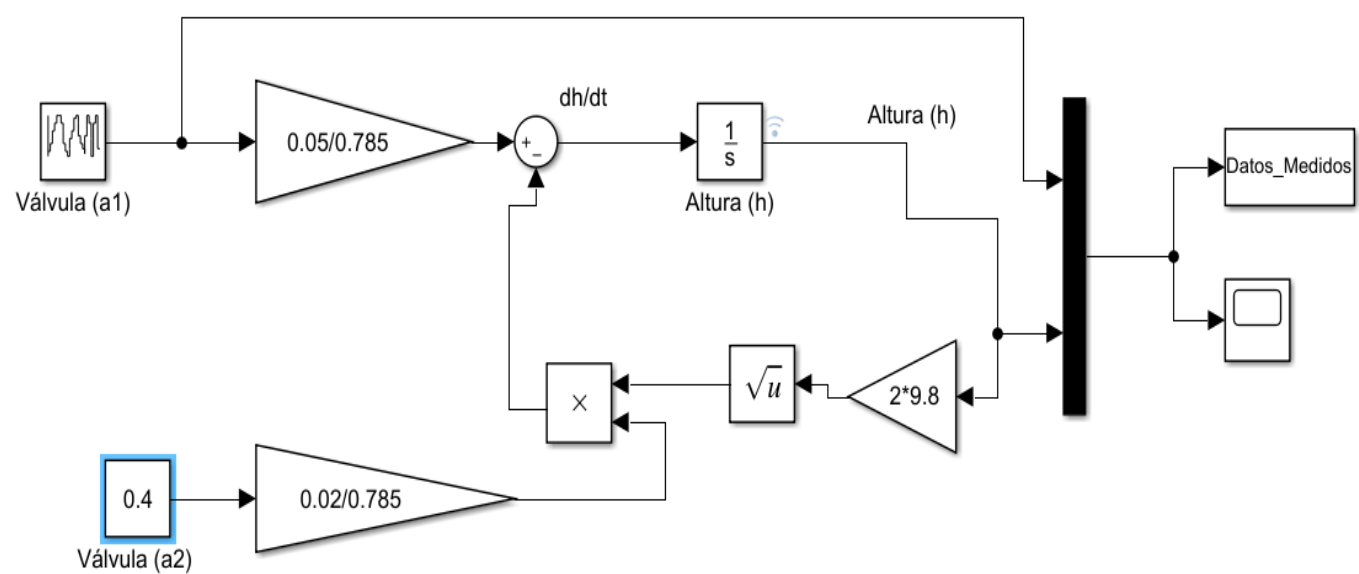


Al sistema de control en lazo abierto de la Figura 6, por medio de una entrada tipo escalón ascendentes y descendentes se manipula a la válvula $a_{1}$ y la válvula $a_{2}$ se mantiene con una apertura de 0.4 (40\%), se almacena la información con una frecuencia de muestreo de 0.1 segundos. En la Figura 7, se presenta la dinámica de la planta de nivel.

\section{Figura 7}

\section{Dinámica del proceso}

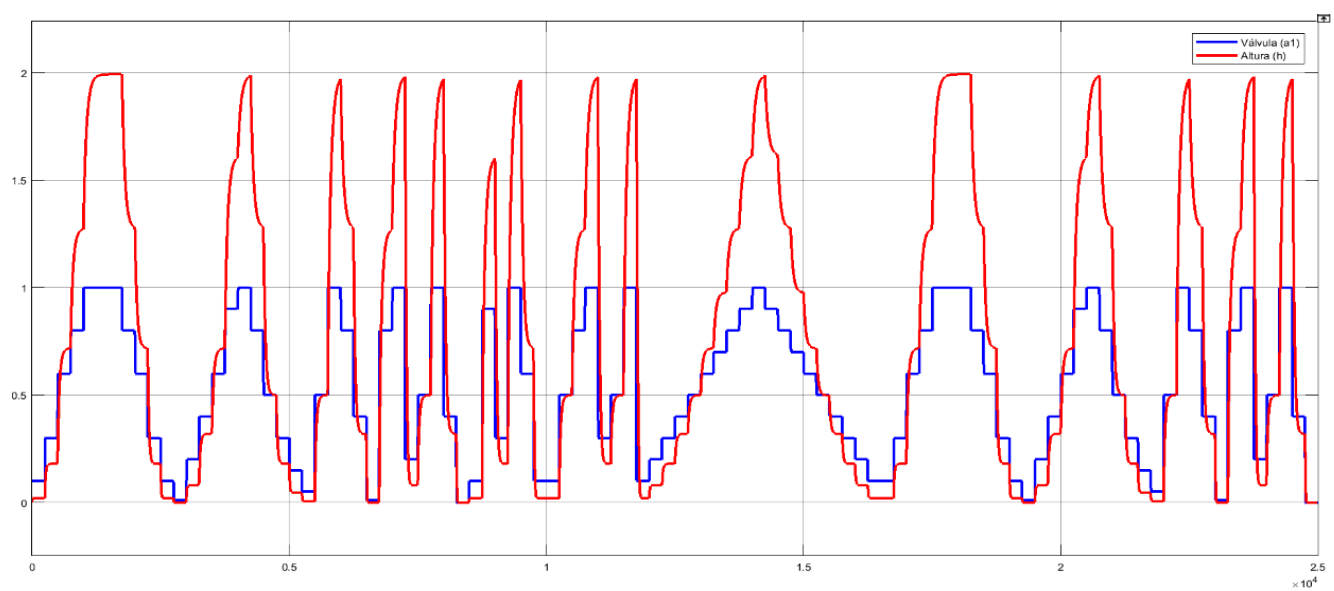

Nota. Entrada: apertura válvula (a1), línea de color azul; Salida: altura (h), línea de color rojo. Número de muestras registradas 25000.

Para identificar el modelo matemático en espacio de estados se hace uso del Toolbox SystemIdentification de Matlab (Troncoso y Suárez, 2017). Con la información registrada de entrada y salida del proceso, se realiza un preprocesamiento segmentando en dos partes: 16500 datos para entrenamiento y 8500 datos para validación. Como se muestra e la Figura 8.

\section{Figura 8}

\section{Preprocesamiento}

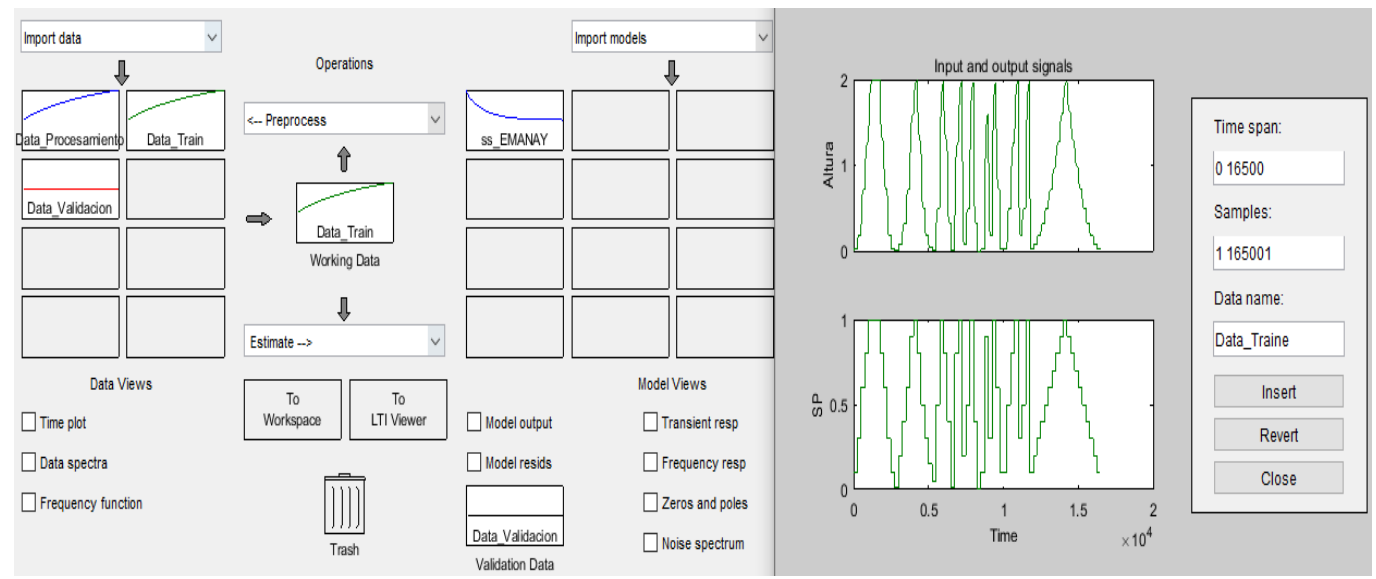


La función de transferencia obtenida en espacio de estados, para el diseño de los controladores obtuvo un $72 \%$ de similitud, se muestra en la Tabla 2.

\section{Tabla 2}

FT en espacio de estados

\begin{tabular}{|c|c|c|c|c|}
\hline \multicolumn{5}{|l|}{$A=$} \\
\hline & $\mathbf{x 1}$ & $\mathrm{x} 2$ & $\mathrm{x3}$ & $x 4$ \\
\hline $\mathbf{x 1}$ & -0.0153 & -0.0189 & $-7.036 \mathrm{E}-06$ & 2.519E-05 \\
\hline $\mathbf{x} 2$ & -0.0135 & -0.016 & -0.0401 & 0.0311 \\
\hline $\mathrm{x} 3$ & -0.138 & -0.1077 & -0.8661 & 3.8831 \\
\hline$x 4$ & 0.1278 & 0.1265 & 0.8274 & -6.3881 \\
\hline \multicolumn{5}{|l|}{$B=$} \\
\hline \multicolumn{5}{|c|}{$\overline{\text { SP }}$} \\
\hline $\mathbf{x 1}$ & \multicolumn{4}{|l|}{0.001052} \\
\hline $\mathbf{x} 2$ & \multicolumn{4}{|l|}{0.008244} \\
\hline $\mathrm{x} 3$ & \multicolumn{4}{|l|}{0.4652} \\
\hline$x 4$ & \multicolumn{4}{|l|}{-0.6948} \\
\hline \multicolumn{5}{|l|}{$\mathrm{C}=$} \\
\hline \multirow[b]{2}{*}{ Altura } & $\mathbf{x 1}$ & $\mathrm{x} 2$ & $x 3$ & $x 4$ \\
\hline & 60.85 & -0.05732 & 0.0001112 & $-1.429 \mathrm{E}-05$ \\
\hline \multicolumn{5}{|c|}{$\mathrm{D}=$} \\
\hline \multicolumn{5}{|c|}{ SP } \\
\hline Altura & 0 & & & \\
\hline
\end{tabular}

\section{Diseño del control Fuzzy}

Se establecen los parámetros y condiciones de funcionamiento adecuados de la planta para el diseño del control Fuzzy. Se establecen 2 entradas y una salida; las variables de entrada son el error $e$, definido como la diferencia entre el valor de altura $h$ deseado y el medido y la derivada del error. El rango o universo de discusión para el error y tasa de cambio del error es de [-1 a 1 ]. La variable de salida del controlador corresponde a la acción de control, cuyo rango es de [-1 a 1].

\section{Conjuntos difusos y variables lingüísticas:}

Se establece siete conjuntos difusos para las variables de entrada; todos los conjuntos están distribuidos uniformemente en todo el universo de discusión mediante funciones de membresía tipo triángulo, a excepción de los extremos que tienen funciones trapezoidales. Puede ver conjuntos borrosos con funciones de pertenencia correspondientes en la Figura 9. 


\section{Figura 9}

Funciones de pertinencia para las variables de entrada

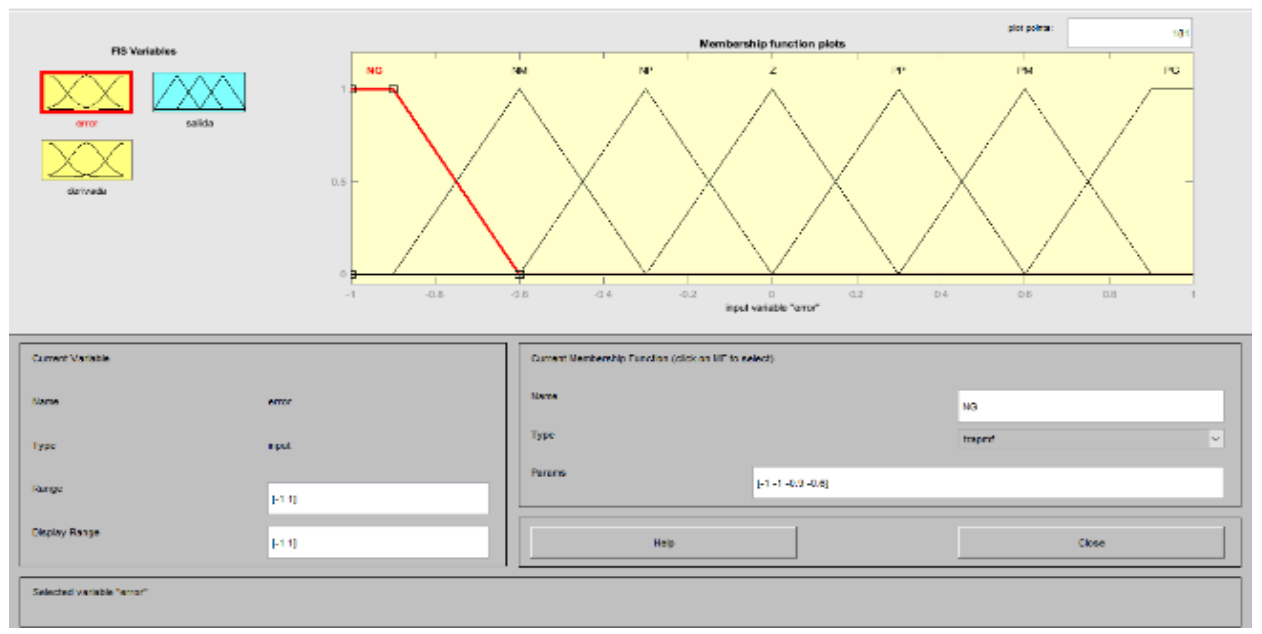

En la Figura 9, se puede apreciar el nombre de las variables lingüísticas asignadas a cada conjunto de entradas, se disponen siete variables: NG (negativo grande), NM (negativo medio), NP (negativo pequeño), Z (cero), PP (positivo pequeño), PM (positivo medio) y PG (positivo grande) (Alomoto Iza y Quimbita Panchi, 2015; Jiménez Madrigal, 2007). Para la variable de salida se establecieron 9 conjuntos difusos distribuidos uniformemente en todo el universo de discusión mediante funciones de membresía tipo triángulo. En la Figura 10, se puede apreciar dichos conjuntos con las respectivas variables lingüísticas asignadas a cada uno: NGG (negativo grande grande), NG (negativo grande), NM (negativo medio), NP (negativo pequeño), Z (cero), PP (positivo pequeño), PM (positivo medio), PG (positivo grande) y PGG (positivo grande grande) (Calle Calle y Guamán Novillo, 2018).

\section{Figura 10}

Funciones de pertinencia para la variable de salida

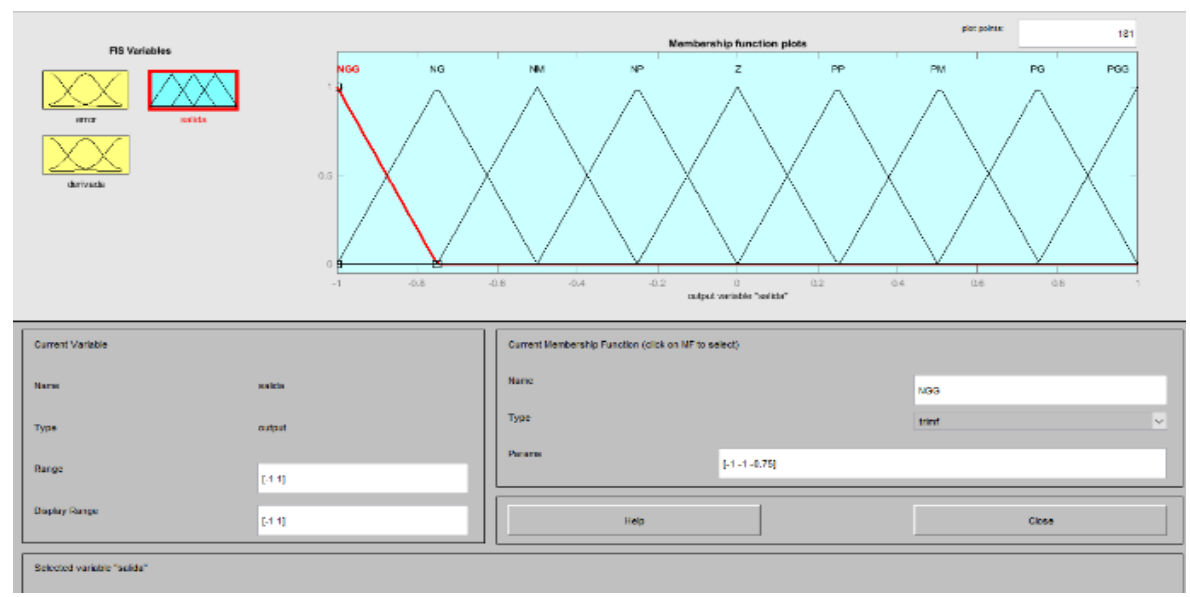




\section{Base de conocimientos y defusificación:}

Una vez que se han definido los conjuntos difusos con las respectivas funciones de pertinencia y variables lingüísticas, se establece el conjunto de 49 reglas de control como se muestra en la Tabla 3.

\section{Tabla 3}

Reglas de control

\begin{tabular}{cccccccc}
\hline e/de & NG & NM & NP & $\mathbf{Z}$ & PP & PM & PG \\
\hline NG & NGG & NGG & NGG & NG & NM & NP & $\mathbf{Z}$ \\
NM & NGG & NGG & NG & NM & NP & $\mathbf{Z}$ & PP \\
NP & NGG & NG & NM & NP & $\mathbf{Z}$ & PP & PM \\
$\mathbf{Z}$ & NG & NM & NP & $\mathbf{Z}$ & PP & PM & PG \\
PP & NM & NP & $\mathbf{Z}$ & PP & PM & PG & PGG \\
PM & NP & $\mathbf{Z}$ & PP & PM & PG & PGG & PGG \\
PG & $\mathbf{Z}$ & PP & PM & PG & PGG & PGG & PGG \\
\hline
\end{tabular}

La Tabla 3, se debe interpretarse con una estructura IF-AND-THEN. Para la defusificación se empleó el método de Mandani, obteniendo la salida mediante el método del centroide (Kouro y Musalem, 2002). En la Figura 11, se presenta las reglas de control.

\section{Figura 11}

Reglas de control en la ventana rule editor

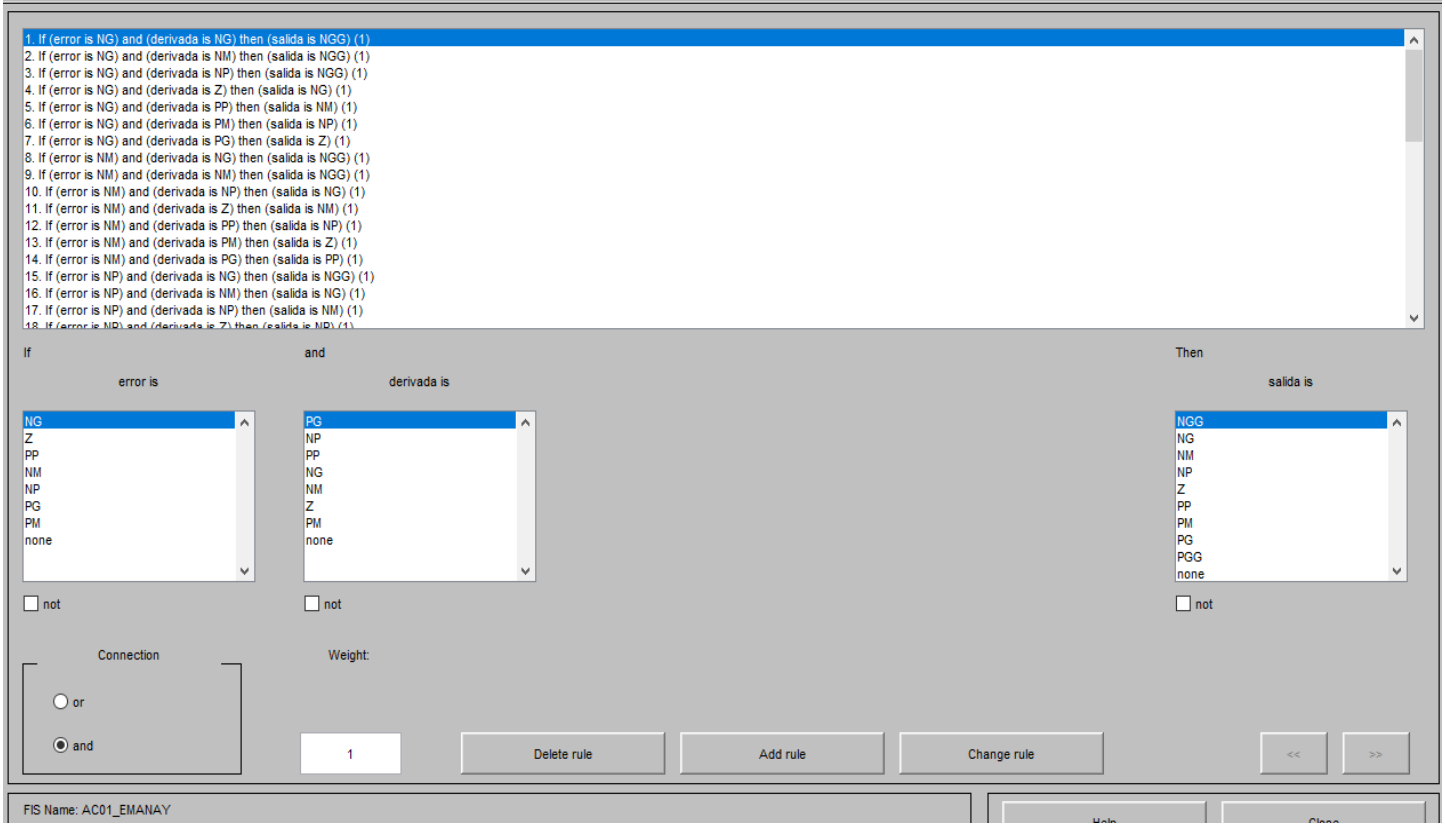




\section{Implementación del controlador Fuzzy en Simulink}

Se implementa el sistema a lazo cerrado con un control PD difuso con acción integral en Simulink, como se muestra en la Figura 12.

\section{Figura 12}

Sistema de control Fuzzy a lazo cerrado

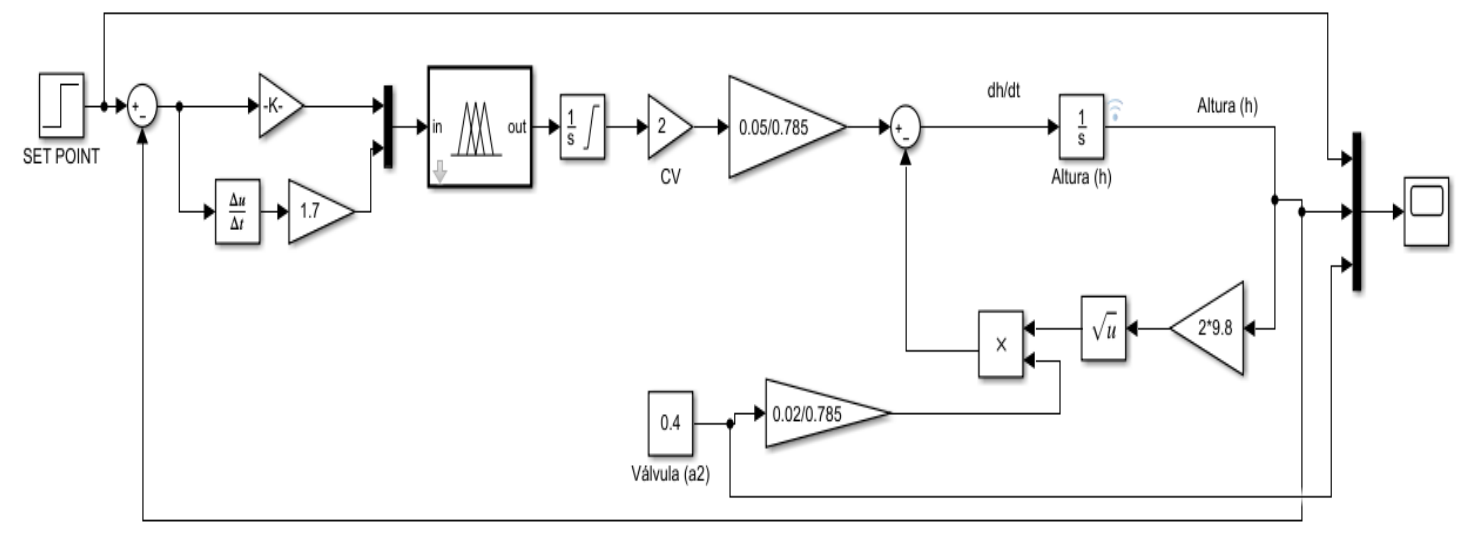

\section{Diseño del control MPC}

\section{Elementos del control predictivo}

El modelo de predicción: debe ser capaz de capturar la dinámica del proceso para poder predecir las salidas futuras (Tsoeu y Esmail, 2011). Para el diseño del MPC, se opta por el modelo en espacio de estados.

Función objetivo: los diferentes algoritmos de control predictivo utilizan diferentes funciones objetivo o de costo para la obtención de la ley de control (Troncoso y Suárez, 2017).

$J=[w(t+1)-\hat{y}(t+1 \mid t)]^{2}+\lambda[\Delta u(t)]^{2}$

Trayectoria de referencia: el sistema reaccionará antes que el cambio haya sido efectivamente aplicado, evitando de este modo retardos en la respuesta del proceso.

\section{Procedimiento}

Obtención del modelo de predicción: Para el diseño del controlador, se basa en el modelo generalizado en espacio de estados (Suwannik et al., 2016).

$\dot{x}(t+1)=A x(t)+B u(t)$

$y(t)=C x(t+1)$

Compresión del optimizador: se usa el siguiente modelo de optimización el mismo que es cuadrático y con restricciones lineales (Jichkar y Sondkar, 2017). 


$$
\begin{aligned}
\operatorname{Min} J(u)=\sum_{k=1}^{N}(\hat{y}(t+k \mid t)-w(t & +k))^{2} \\
u m i ́ n & \leq u \leq u m a ́ x \\
0 & \leq u \leq 1 \\
y \text { mín } & \leq y \leq y \text { máx } \\
0 & \leq y \leq 1
\end{aligned}
$$

Parámetros de sintonización: se establece un tiempo de muestreo de 0.1 segundos, horizonte de predicción de 150 y horizonte de control de 15 (Báez Pedraza, 2018).

\section{Implementación del controlador en Simulink}

Para la simulación se propone el diagrama de control diseñado en Simulink, nótese que se ha desactivado la medición de perturbación, puesto que no está considerado en el modelamiento dicha variable, Figura 13, el bloque del controlador predictivo está configurado como ilustra la Figura 14.

Figura 13 Diagrama de control MPC

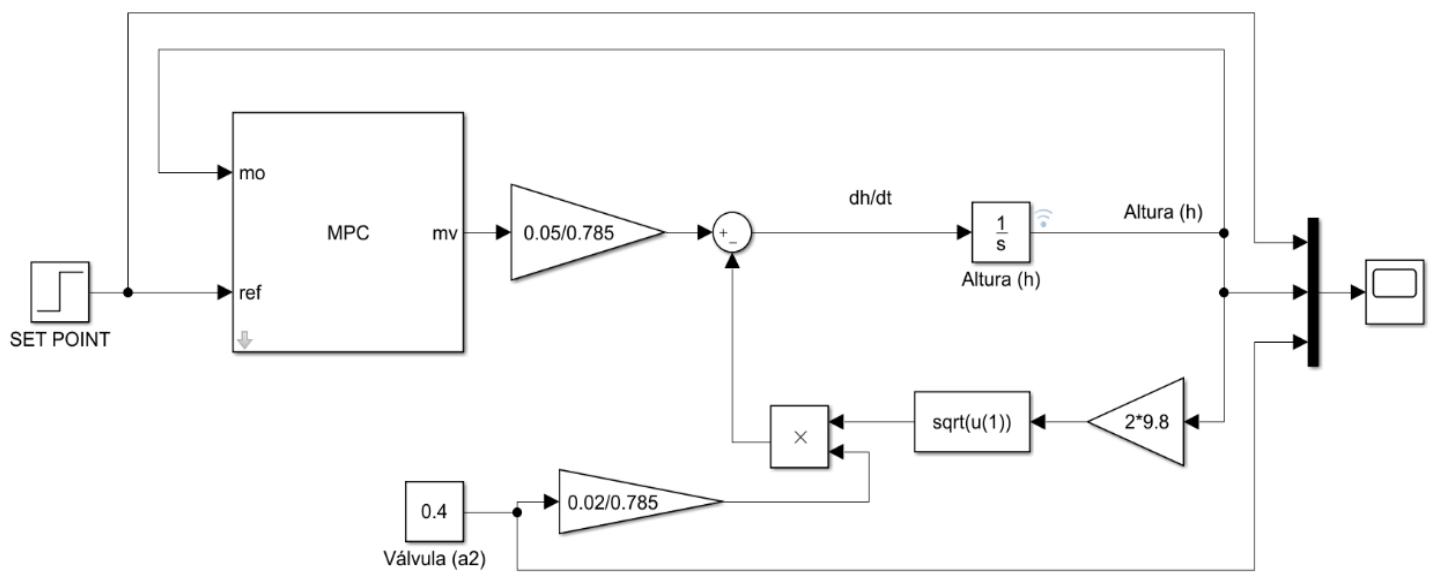

Figura 14 Configuración del control MPC

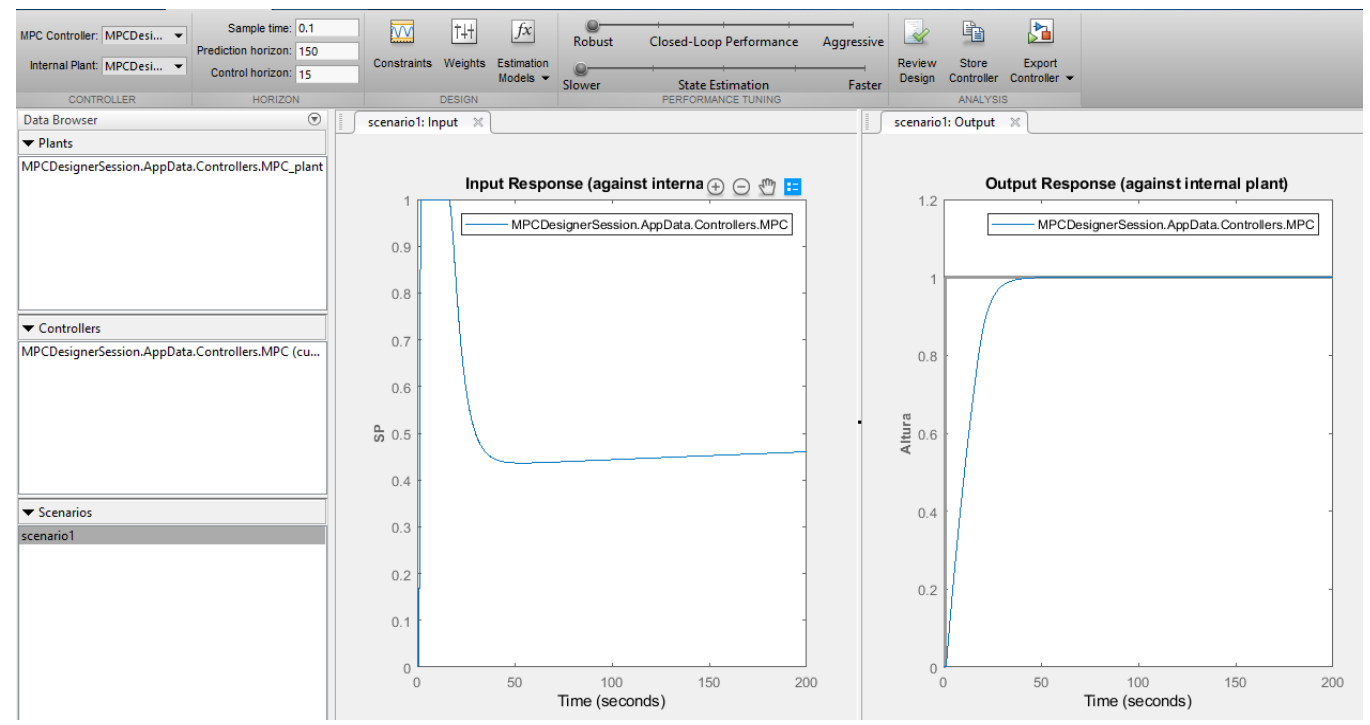




\section{RESULTADOS Y DISCUSIÓN}

El sistema de control PD difuso con acción integral y MPC, para la simulación en base al diseño de la Figura 13 y Figura 14 respectivamente, se sometió a una entrada de tipo escalón unitaria, con una apertura de la válvula $a_{2}$ de [0.4 a 0.56$]$, se verifico el comportamiento del sistema frente al controlador PID.

Se presenta cada uno de los resultados en la Figura $(15,16,17)$.

Nota. Señal del controlador, línea de color verde; Variable de proceso: nivel, línea de color azul; Set Point: válvula $a_{1}$, línea de color rojo; Apertura Válvula $\left(a_{2}\right)$, línea de color naranja.

\section{Figura 15}

\section{Control PID}

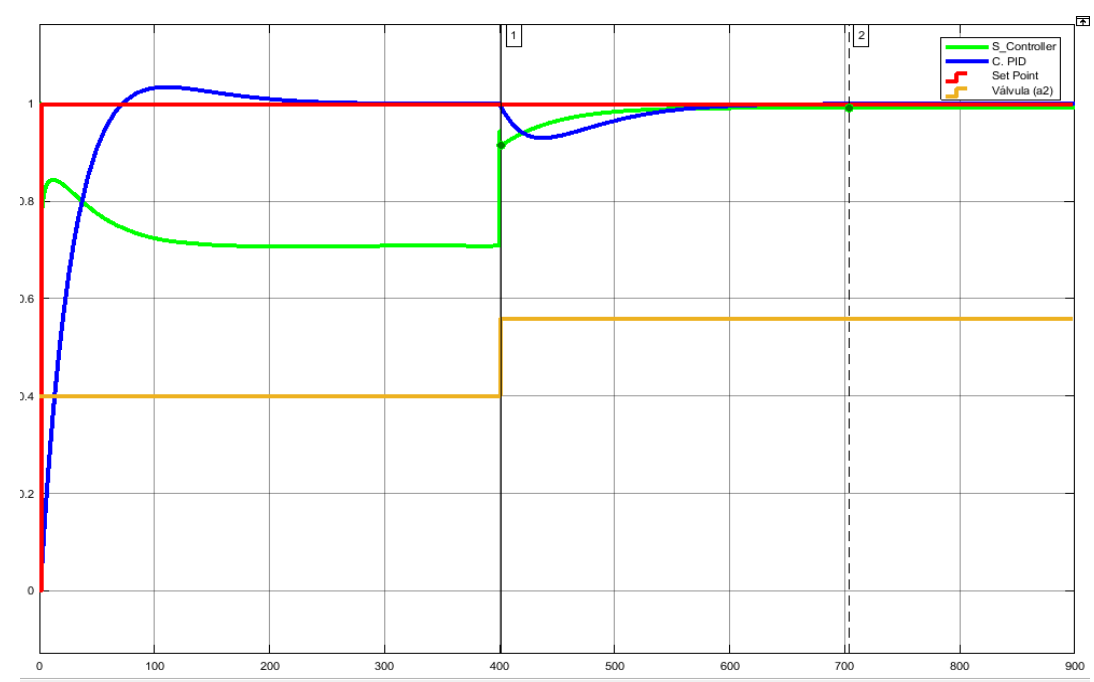

\section{Figura 16}

Control PD difuso con acción integral

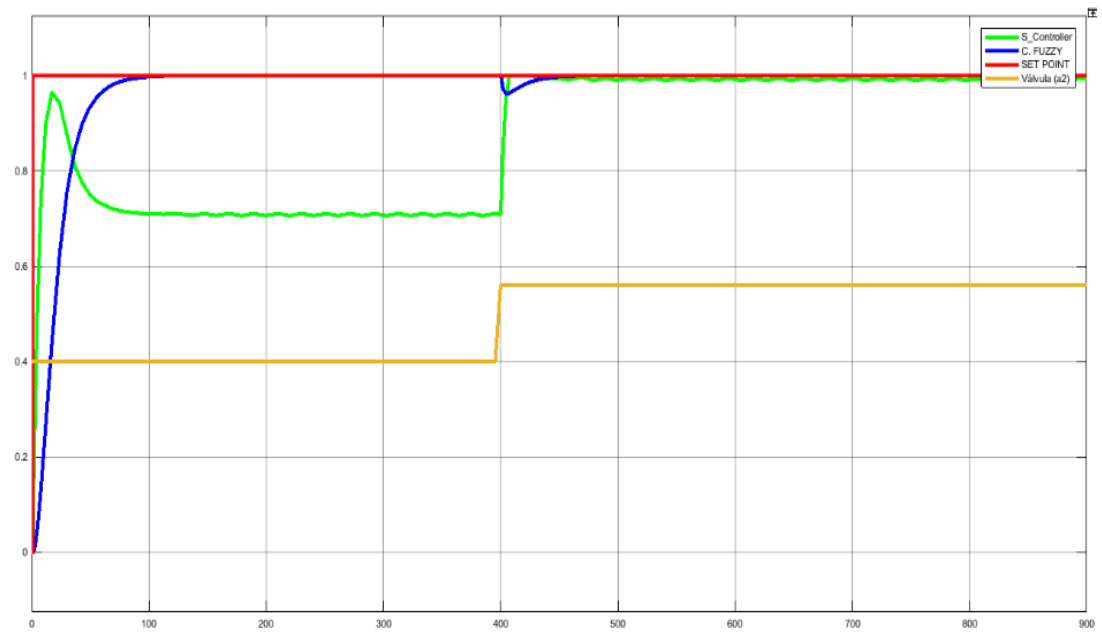




\section{Figura 17}

Control MPC

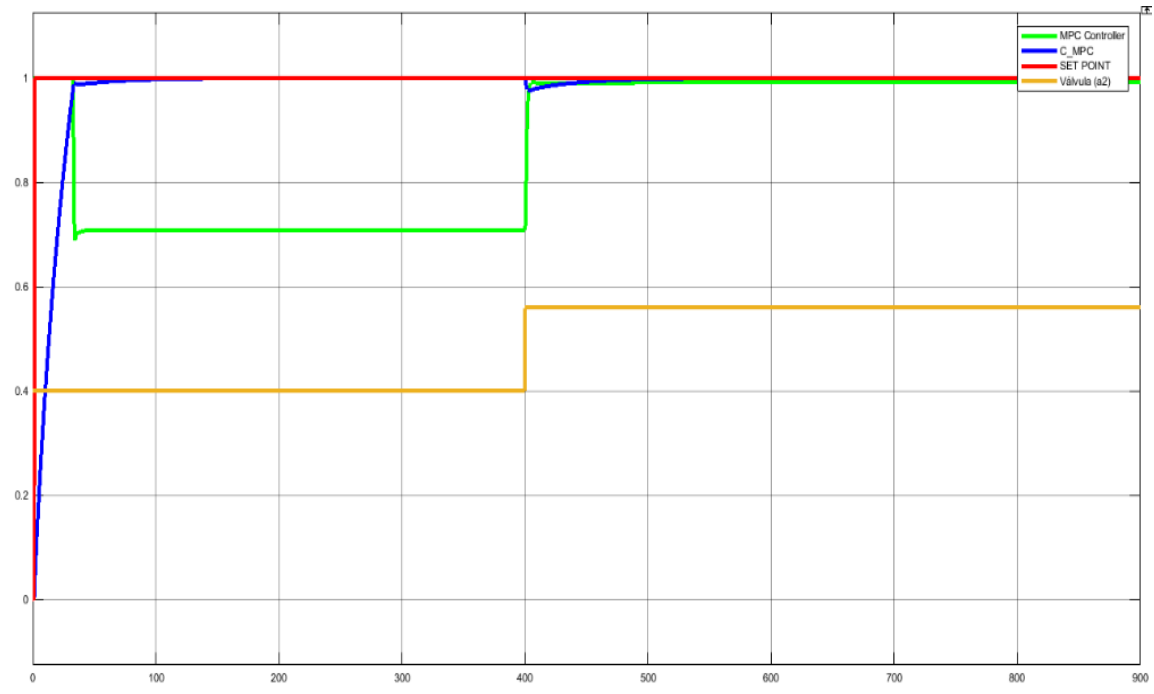

De las Figuras $(15,16,17)$, se extraen características representativas como se muestran en la Tabla 4.

\section{Tabla 4.}

Características de control PID, Fuzzy y MPC con apertura de la válvula $a_{2}=0.4$

\begin{tabular}{|c|c|c|c|c|c|c|c|}
\hline \multicolumn{5}{|c|}{ Tiempo (s) } & \multicolumn{3}{|c|}{ Tiempo (s) } \\
\hline & $\begin{array}{c}\text { Retardo } \\
\left(t_{d}\right)\end{array}$ & $\begin{array}{c}\text { Crecimiento } \\
\left(t_{r}\right)\end{array}$ & $\begin{array}{l}\text { Pico } \\
\left(t_{p}\right)\end{array}$ & $\begin{array}{c}\text { Sobre } \\
\text { impulso } \\
\text { máximo } \\
\quad\left(M_{p}\right)\end{array}$ & $\begin{array}{c}\text { Establecimiento } \\
\qquad\left(t_{s}\right)\end{array}$ & $\begin{array}{l}\text { Variable } \\
\text { de } \\
\text { proceso: } \\
\text { nivel del } \\
\text { líquido } \\
\text { (h) }\end{array}$ & $\begin{array}{c}\text { Error en } \\
\text { estado } \\
\text { Estacionario }\end{array}$ \\
\hline PID & 18.262 & 71.644 & 101.613 & $3.30 \%$ & 292.664 & 1.001 & $0.20 \%$ \\
\hline $\begin{array}{c}\text { Fuzz } \\
\mathrm{y}\end{array}$ & 20.135 & 84.755 & 0 & 0 & 119.407 & 0.9991 & $0.09 \%$ \\
\hline MPC & 13.580 & 51.977 & 0 & 0 & 140.947 & 0.9999 & $0.01 \%$ \\
\hline
\end{tabular}




\section{Tabla 5.}

Características de control PID, Fuzzy y MPC con apertura variable de la válvula $a_{2}=$ [0.4 a 0.56 ]

\begin{tabular}{cccccc}
\hline & Tiempo de & Tiempo & & \multicolumn{3}{c}{$\begin{array}{c}\text { Variable } \\
\text { de } \\
\text { perturbación } \\
\text { Transitorio }\end{array}$} & $\begin{array}{c}\text { Tiempo de } \\
\text { establecimiento } \\
\left(\boldsymbol{t}_{\boldsymbol{s}}\right)\end{array}$ & $\begin{array}{c}\text { proceso: } \\
\text { nivel del } \\
\text { líquido } \\
(\mathbf{h})\end{array}$ & $\begin{array}{c}\text { Error en } \\
\text { estado } \\
\text { Estacionario }\end{array}$ \\
\hline PID & 400 & 167.638 & 244.433 & 0.9982 & $0.20 \%$ \\
Fuzzy & 400 & 35.588 & 82.414 & 0.9989 & $0.09 \%$ \\
MPC & 400 & 46.826 & 137.669 & 0.9999 & $0.01 \%$ \\
\hline
\end{tabular}

En base a las Tablas 4, 5: el controlador PID, genera un sobre impulso máximo de 3.30 $\%$, un tiempo de establecimiento de 292.664 segundos y un error de estado estacionario de $0.20 \%$. El control Fuzzy por su parte no genera sobre impulso, con un tiempo de estabilización de 119.407 segundos con un error en estado estacionario de $0.09 \%$. La respuesta del controlador MPC es rápida con un tiempo de establecimiento de 140.947 segundos y no tiene sobre impulso máximo, no obstante, existe un cambio brusco de la parte transitoria a la parte estable con un error en estado estacionario de $0.01 \%$.

Los controladores diseñados ante una la apertura de la válvula $a_{2}$ menores a 0.56 (56\%), tienen la capacidad de volver a estabilizarse, mientras que, si ingresamos una perturbación a la válvula $a_{2}$ mayor a 0.6 (60\%), ya no tienen la capacidad de volver a recuperarse.

\section{CONSIDERACIONES FINALES}

El control Fuzzy: PD difuso con acción integral no genera sobre impulso, con un tiempo de estabilización de 119.407 segundos y un error en estado estacionario de $0.09 \%$.

La respuesta del controlador MPC es rápida con un tiempo de establecimiento de 140.947 segundos y no tiene sobre impulso máximo, pero no obstante existe un cambio brusco de la parte transitoria a la parte estable con un tiempo de crecimiento de 51.977 segundos y un error en estado estacionario del $0.01 \%$.

El control Fuzzy: PD difuso con acción integral, presenta un mejor rendimiento para el proceso de nivel, ya que, el tiempo de establecimiento es de 119.407 segundos y ante una perturbación se establece en un tiempo de 82.414 segundos. 
Los controladores Fuzzy, MPC y PID tienen un buen desempeño ante aberturas de la válvula $a_{2}$ no mayores del $56 \%$.

\section{LISTA DE REFERENCIAS}

Alomoto Iza, L. M., \& Quimbita Panchi, E. A. (2015). Artículo Científico-Diseño e implementación de controladores difusos aplicado a los procesos de Presión y Flujo del Laboratorio de Redes Industriales y Control de Procesos de la Universidad de las Fuerzas Armadas-ESPE Extensión Latacunga, integrados por una Red Ethernet Industrial. http://repositorio.espe.edu.ec/handle/21000/9346

Alotaibi, M., Balabid, M., Albeladi, W., \& Alharbi, F. (2019, June). Implementation of liquid level control system. In 2019 IEEE International Conference on Automatic Control and Intelligent Systems (I2CACIS) (pp. 311-314). IEEE. doi:10.1109/i2cacis.2019.8825058

Åström, K. J., \& Murray, R. M. (2008). Feedback systems. An Introduction for Scientists and engineers. Princeton University Press.

Báez Pedraza, M. C. (2018). Control predictivo multivariado para el sistema de péndulo invertido. Bogotá. [Tesis de Ingeniería, Universidad Santo Tomas]. https://repository.usta.edu.co/jspui/bitstream/11634/15462/1/2019camilabaez.pdf

Bo, B., Chuang, L., \& Meng, C. (2016, July). Based on plc fuzzy control algorithm in the application of level control. In 2016 International Symposium on Computer, Consumer and Control (IS3C) (pp. 698-701). IEEE. doi:10.1109/is3c.2016.179

Calle Calle, D. E., \& Guamán Novillo, P. F. (2018). Diseño e implementación de un control difuso de velocidad para el motor hidráulico HRE HIDRAULIC [Tesis de ingeniería, Universidad Politécnica Salesiana].

https://dspace.ups.edu.ec/bitstream/123456789/16369/1/UPS-CT007970.pdf

De Prada, C. (2010). El futuro del control de procesos. Revista Iberoamericana de automática e informática industrial, 1(1), 5-14. doi: 10.4995/riai.2004.8020

Efheij, H., Albagul, A., \& Albraiki, N. A. (2019, March). Comparison of model predictive control and PID controller in real time process control system. In 2019 19th International Conference on Sciences and Techniques of Automatic Control and Computer Engineering (STA) (pp. 64-69). IEEE. doi:10.1109/sta.2019.8717271

Gehlaut, S., Varshney, T., \& Gupta, S. (2018, June). Performance Analysis of MPC for Level Control of Modified Quadruple Tank System. In 2018 2nd International 
Conference on Power, Energy and Environment: Towards Smart Technology (ICEPE) (pp. 1-6). IEEE. doi:10.1109/epetsg.2018.8659317

Jichkar, C. D., \& Sondkar, S. Y. (2017, April). Comparative study of real time implementation of LabVIEW based MPC controller and PID controller for flow control loop. In 2017 2nd International Conference for Convergence in Technology (I2CT) (pp. 464-470). IEEE. doi:10.1109/i2ct.2017.8226172

Jiménez Madrigal, R. (2007). Diseño de un controlador lógico difuso, aplicado al control de posición de un servomotor de $\mathrm{CD}$, usando un algoritmo genético. https://cdigital.uv.mx/bitstream/handle/123456789/1736/Tesis-Jimenez-MadrigalRene.pdf? sequence $=1 \&$ isAllowed $=\mathrm{y}$

Kouro, S., \& Musalem, R. (2002). Control mediante lógica difusa. Técnicas Modernas en Automática, 1, 1-7. http://www2.elo.utfsm.cl/ elo377/documentos/Fuzzy.pdf

Mursyitah, D., Faizal, A., \& Ismaredah, E. (2018, October). Level Control in Coupled Tank System Using PID-Fuzzy Tuner Controller. In 2018 Electrical Power, Electronics, Communications, Controls and Informatics Seminar (EECCIS) (pp. 293-298). IEEE. doi:10.1109/eeccis.2018.8692846

Patel, H. R., \& Shah, V. (2018, May). Fuzzy logic based passive fault tolerant control strategy for a single-tank system with system fault and process disturbances. In 2018 5 th international conference on electrical and electronic engineering (ICEEE) (pp. 257-262). IEEE. doi:10.1109/iceee2.2018.8391342

Perez, M., Pérez, A., \& Pérez, E. (2008). Introducción a los sistemas de control y modelo matemático para sistemas lineales invariantes en el tiempo. Universidad Nacional de San Juan. http://dea.unsj.edu.ar/control1/apuntes/unidad1y2.pdf

Popescu, M. (2018). Comparative study of PID and Fuzzy level control using delta v distributed system. In 2018 10th International Conference on Electronics, Computers and Artificial Intelligence (ECAI) (pp. 1-4). IEEE. doi:10.1109/ecai.2018.8679011

Prusty, S. B., Pati, U. C., \& Mahapatra, K. (2014, February). Implementation of fuzzyPID controller to liquid level system using LabVIEW. In Proceedings of the 2014 International Conference on Control, Instrumentation, Energy and Communication (CIEC) (pp. 36-40). IEEE. doi:10.1109/ciec.2014.6959045

Suwannik, P., Jennawasin, T., \& Banjerdpongchai, D. (2016, November). Design of linear model predictive control for level control process with output feedback from 
wireless transmitter. In 2016 International Automatic Control Conference (CACS) (pp. 132-136). IEEE. doi:10.1109/cacs.2016.7973897

Troncoso, C., \& Suárez, A. (2017). Control del Nivel de Pulpa en un Circuito de Flotación utilizando una Estrategia de Control Predictivo. Revista Iberoamericana de Automática e Informática industrial, 14(3), 234-245. doi: 10.1016/j.riai.2017.04.003

Tsoeu, M. S., \& Esmail, M. (2011, September). Unconstrained MPC and PID evaluation for motion profile tracking applications. In IEEE Africon'11 (pp. 1-6). IEEE. doi:10.1109/afrcon.2011.6072037

Tzafestas, S., \& Papanikolopoulos, N. P. (1990). Incremental fuzzy expert PID control. IEEE Transactions on Industrial Electronics, 37(5), 365-371. doi: $10.1109 / 41.103431$

Vinothkumar, C., \& Esakkiappan, C. (2021). Fuzzy PI and Fuzzy PID Controller Based Hopper Tank Level Control System. In 2021 International Conference on Advances in Electrical, Computing, Communication and Sustainable Technologies (ICAECT) (pp. 1-5). IEEE. doi:10.1109/icaect49130.2021.9392451

Yao, G., Gao, F., Wang, C., \& Chen, X. (2009, June). Design and simulation based on Kalman filter fuzzy adaptive PID control for mold liquid level control system. In 2009 Chinese Control and Decision Conference (pp. 6105-6109). IEEE. doi:10.1109/ccdc.2009.5195297 